\title{
GREEN BONDS AS A FINANCE TOOL OF RENEWABLE ENERGY
}

\author{
DOI: 10.17261/Pressacademia.2021.1516 \\ PAP- V.14-2021(42)-p.152-153
}

\section{Meryem Filiz Basturk}

Bursa Uludag University, Department of Economics, Bursa, Turkey. meryemfiliz@uludag.edu.tr, ORCID: 0000-0002-9085-2295

\section{To cite this document}

Basturk, M. F., (2021). Green bonds as a finance tool of renewable energy. PressAcademia Procedia (PAP), 14, 152-153.

Permanent link to this document: http://doi.org/10.17261/Pressacademia.2021.1516

Copyright: Published by PressAcademia and limited licensed re-use rights only.

\begin{abstract}
Purpose- Nowadays, green bonds are essential tools for renewable energy finance. The increasing damage caused by fossil fuels to the environment increases the importance of renewable energy investments in both developed and developing economies. Although renewable energy investments are increasing, the fact that initial costs are higher than fossil resources make the issue of how to finance investments important. Because high costs make it difficult to find a financing source, this study examines the development of green bonds, which is one of these tools.

Methodology- For economies to achieve sustainability goals, it is clear that the field of green bonds will maintain its vitality in the coming years. The number of studies on green bonds is increasing nowadays. In this study, green bonds will be discussed with a detailed literature review.

Findings- Green bonds, which are one of the tools used in financing renewable energy, have become increasingly widespread in the world. The European Investment Bank first issued green bonds in 2007. In 2007, 600 million Euros of green bonds were issued, and by 2019, this figure increased to 271 billion \$. At first, while multilateral banks are more dominant in the issuance channels of bonds, depending on the increasing interest. It has been seen that companies, national governments and financial institutions show interest in bond issuance.

Conclusion- Considering the global climate change, the damage caused by fossil wastes to the environment and the sustainable growth targets of the economies, it can be stated that the importance of green bond issuance will increase in the future.
\end{abstract}

Keywords: Green bonds, renewable energy, multilateral development banks, developed countries, developing countries JEL Codes: Q20, 013, 044

\section{YENILENEBILIR ENERJININ FINANSMAN ARACI OLARAK YEȘiL TAHVILLER}

\section{ÖZET}

Amaç- Yeşil tahviller, günümüzde yenilebilir enerji kaynaklarının finansmanın önemli bir aracıdır. Fosil atıkların çevreye verdiği zararların giderek artması hem gelişmiş hem gelişmekte olan ekonomilerde yenilenebilir enerji yatırımlarının önemini arttırmaktadır. Her ne kadar yenilenebilir enerji yatırımları artsa da başlangıç maliyetlerinin fosil kaynaklara göre yüksek olması, yatırımların finansmanının nasıl sağlanacağını konusunu önemli kılar. Zira yüksek maliyetler finansman kaynağı bulunmasını güçleştirir. Bu çalışmanın amacı bu araçlardan biri olan yeşil tahvillerin gelişimini incelemektir.

Yöntem- Ekonomilerin sürdürülebilirlik hedeflerini gerçekleştirmek için ilerleyen yıllarda yeşil tahviller ile ilgili alanın canlılı̆ını koruyacağı açıktır. Günümüzde yeşil tahvilleri konu edinen araştırmaların sayısı artış göstermektedir. Bu çalışmada, yeşil tahvillerin ilk çıkarılışından günümüze kadar izlemiş olduğu seyir ayrıntılı bir literatür taraması ile ele alınmaya çalışılacaktır.

Bulgular- Yenilenebilir enerjinin finansmanında kullanılan araçlardan biri olan yeşil tahviller dünya ölçeğinde giderek yaygınlaşmaktadır. Yeşil tahviller ilk olarak 2007'de Avrupa Yatırım Bankası tarafından ihraç edilmiştir. 2007 yılında 600 milyon Euro yeşil tahvil ihraç edilmiş, 2019 yılına gelindiğinde ise bu rakam 271 milyar \$’a çıkmıştır. Başlangıçta tahvillerin ihraç kanallarında daha fazla çok taraflı kalkınma bankalarının ağırlığı söz konusuyken; artan ilgiye bağlı olarak ihraç kanalları çeşitlenmiştir. Şirketlerin, ulusal hükümetlerin ve finansal kuruluşların tahvil ihracına ilgi gösterdiği görülmüştür.

Sonuç- Küresel iklim değişikliği, fosil atıkların çevreye verdiği zarar ve ekonomilerin sürdürülebilir büyüme hedefleri göz önüne alındığında yeşil tahvil ihracının ilerleyen dönemde öneminin giderek artacağı belirtilebilir.

Anahtar Kelimeler: Yeşil tahviller, yenilenebilir enerji, çok taraflı kalkınma bankaları, gelişmiş ülkeler, gelişmekte olan ülkeler JEL Kodları: Q20, 013, O44 


\section{REFERENCES}

Bitlis, M. (2016). Green bonds. Escarus Blog, http://www.escarus.com/green-bonds, (Erişim Tarihi: 01.20.2019).

CBI (2021). Sustainable Debt Global State of the Market 2020, Climate Bonds Initiative,

https://www.climatebonds.net/resources/reports/sustainable-debt-global-state-market-2020, (Erişim Tarihi: 29.09.2020).

CBI (2020a). 2019 Green Bond Market Summary, Climate Bonds Initiative, https://www.climatebonds.net/resources/reports/2019-greenbond-market-summary, (Erişim Tarihi: 29.09.2020).

CBI (2020b). Green Bonds Global State of the Market 2019, Climate Bonds Initiative,

https://www.climatebonds.net/resources/reports/green-bonds-global-state-market-2019 (Erişim Tarihi: 29.09.2020).

CBI (2017). Bonds and Climate Change the State of the Market 2017, Climate Bonds Initiative, https://www.climatebonds.net/files/reports/cbi-sotm_2017-bondsclimatechange.pdf, (Erişim Tarihi: 01.08.2020).

Griffith-Jones, S., Ocampo, J.A. \& Spratt, S. (2012). Financing Renewable Energy in Developing Countries: Mechanisms and Responsibilities. European Report on Development,

http://policydialogue.org/files/publications/Financing_Renewable_Energy_in_Developing_Countries.pdf, (Erişim Tarihi: 18.11.2020)

IDFC [International Development Finance Club] (2014). Scaling up Renewable Energy Investments.

https://www.idfc.org/Downloads/Publications/02_other_idfc-expert_documents/IDFC_Sustainable_Infrastructure_Paper_01-12-14.pdf, (Erişim Tarihi: 25.07.2018).

IRENA (2020). Renewable Energy Finance: Green Bonds, International Renewable Energy Agency, Abu Dhabi, https://www.irena.org/publications/2020/Jan/RE-finance-Green-bonds, (Erişim Tarihi: 02.03.2021).

IRENA and CPI (2020). Global Landscape of Renewable Energy Finance, International Renewable Energy Agency, Abu Dhabi, https://www.irena.org/publications/2020/Nov/Global-Landscape-of-Renewable-Energy-Finance-2020, (Erişim Tarihi: 07.05.2021).

Kandır, S. Y., \& Yakar, S. (2017). Yenilenebilir enerji yatırımları için yeni bir finansal araç: Yeşil tahviller, Maliye Dergisi, 172, 85-110.

Kaygusuz, K. (2007). Energy for sustainable development: Key issues and challenges, Energy Sources, Part B: Economics, Planning, and Policy, 2(1), 73-83.

Lam, P., \& Law, A. (2018). Financing for renewable energy projects: A decision guide by developmental stages with case studies. Renewable and Sustainable Energy Reviews, 90, 937-944.

Müller, S., Brown, A., \& Ölz, S. (2011). Renewable energy policy considerations for deploying renewables. Information Paper. IEA. https://www.iea.org/publications/freepublications/publication/Renew_Policies.pdf, (Erişim Tarihi: 10 Haziran 2019).

Philibert, C. (2011). Interactions of policies for renewable energy and climate. Working Paper, IEA, https://www.oecdilibrary.org/docserver/5kggc12rmkzq-

en.pdf?expires=1527756748\&id=id\&accname=guest\&checksum=E7B1DF8C7058B42939CD1A5A11B1ACC9, (Erişim Tarihi: 05.05.2021)

SNB (2018). Creating Green Bond Markets Insights, Innovations and Tools from Emerging Markets,

https://www.climatebonds.net/resources/reports/creating-green-bond-markets-insights-innovations-and-tools-emerging-markets, (Erişim Tarihi: 05.05.2021)

UNEP (2020). Global Trends in Renewable Energy Investment 2020, https://www.fs-unep-centre.org/wpcontent/uploads/2020/06/GTR_2020.pdf, (Erişim Tarihi: 08.07.2021). 